\title{
" THE TWO OLD BACHELORS " DE EDWARD LEAR: ÉTUDE SÉMANTIQUE ET TRADUCTOLOGIQUE
}

\section{INTRODUCTION}

Cet article a pour ambition de saisir du point de vue de la sémantique interprétative un court-texte poétique de Edward Lear intitulé "The two old bachelors " ${ }^{1}$ (tiré de Laughable lyrics, a fourth book of nonsense poems, songs, botany, music, etc, 1877) traduit en français exactement un siècle plus tard par Patrick Hersant (Edward Lear Nonsense, 1977, éd. Ombres). Outre une analyse proprement sémantique de la version originale anglaise du poème et de ce qui en fait un texte absurde, nous souhaitons montrer sur la base de cette analyse préparatoire comment Patrick Hersant a tranché le dilemme traductologique auquel il a été confronté : en effet, une partie de l'absurde reposant sur l'homonymie en anglais, le traducteur a livré une interprétation très peu fidèle à l'original, peut être volontairement.

Les critiques ont pu évoquer l'œuvre de Edward Lear selon plusieurs angles, souvent de manière concomitante : le fait que l'auteur ait été contemporain de Lewis Carroll, son ancrage spatio-temporel dans l'Angleterre de l'époque victorienne, son homosexualité à la fois connue mais tue. Ces angles d'approche constituent, à propos du poème «The two old bachelors », une grille de lecture aussi commode qu'insuffisante, en ce sens qu'ils ne délivrent aucune piste de détermination de ce qui fait le nonsense de ce texte en soi.

« The two old bachelors » est ce que Petr Levi (2013 : 299) appelle un « desultary tale », c'est-à-dire littéralement un conte décousu. Le texte raconte l'histoire de deux vieux hommes (dont il n'est pas sûr qu'ils soient célibataires comme le dira Hersant) vivant ensemble dans un dénuement proche de l'extrême pauvreté, puisqu'ils n'ont plus rien à manger. Ce fait étant posé d'emblée, les deux hommes se lancent dans une chasse

1 Nous laissons tant le texte original que la traduction française en annexes. 
peu fructueuse : l'un ne récolte qu'un muffin et l'autre une souris. Malgré tout, pour cuisiner ce maigre butin, il semble leur falloir un accompagnement digne de ce nom, une « farce » désignée en anglais par «stuffin ». C'est alors que les choses se compliquent car les deux hommes, pour ce faire, se mettent en quête de « sage and onion », littéralement en français « sauge et oignons » : on verra que le lexème " sage » anglais est justement le nœud du problème, car il peut aussi désigner dans la langue de Shakespeare un « homme sage ». Les deux hommes trouvent bien deux gros oignons - seulement empruntés («borrowed ») d'ailleurs, mais pas de sauge : un villageois de mauvais conseil leur indique ainsi qu'il existe bien un sage qui lit un livre à longueur de journée en haut d'une vallée, ce sur quoi les deux hommes se mettent à sa recherche. Alors qu'ils parviennent à sa hauteur après une raide ascension, les deux hommes préviennent le sage que c'en est fini pour lui et qu'il va devoir finir en farce ; mais ceux-ci sont assommés par le sage avec le livre en question (cf. illustration en annexes) : ils dégringolent tout en bas jusqu'à leur maison où la souris a mangé le muffin avant de s'enfuir. On apprend enfin, comme c'est souvent le cas dans les limericks de Lear, que l'on n'entendit plus parler d'eux et qu'on ne sait pas même ce qu'ils sont devenus.

\section{1 "The two old bachelors": une analyse biographique (homo)sexuelle}

Ce texte a donné lieu à des interprétations pertinentes et complémentaires mais toutes se rejoignent sur un point, ce qu'on pourrait appeler une interprétation (homo)sexuelle : selon cette perspective, on peut estimer que les deux vieillards ne sont pas satisfaits de leur relation et cherchent de fait un troisième homme. Cette piste interprétative s'appuie sur les indices suivants : 'earnest' qui qualifie « sage » dans le poème et qui semble avoir été un adjectif codé pour désigner les homosexuels à l'époque victorienne, s'inscrivant dans le paradigme des formules euphémiques et de contournement de la politesse feutrée victorienne ; on peut aussi cerner dans les deux gros oignons, le sommet perpendiculaire et le crâne chauve des vieillards une métaphore phallique peut-être moins évidente. D'après Matthew Bevis (2016 : 145) qui s'intéresse particulièrement aux chutes (dans leur sens littéral) chez Lear, cette interprétation doit être soutenue par les vers finaux " they rolled promiscuous down » et en particulier l'idée selon laquelle « the precise nature of that promiscuity is left open ». Selon Peter Robinson (2016 : 127), la farce ou « stuffin » est l'indice interprétatif central et cette visée cannibale n'est autre que ce qu'il appelle des « 'inappropiate appetites', the form of love, for instance, that dared not speak its name ».

Il est clair que, l'isotopie /sexualité/ confère au genre de la recette de cuisine ponctuellement affirmé dans le poème, un statut parodique en montrant ce qui arrive lorsqu'on fait une erreur dans la recette : c'est ce qui fait dire à Peter Robinson (2016:126) que 
tout le poème rapporte « what can go wrong when you try to follow a recipe but you don't have the right ingredients ».

Quoiqu'il en soit, cette recette, aussi bien déclinée dans la version française (cf. « réservez ») que dans le texte original, peut être perçue comme le code de la société victorienne. Le fait qu'elle soit incomprise et mal suivie, si on peut le dire ainsi, constitue l'assurance d'une mise au ban de la société, ce que Lear craignait très fortement : or toute affirmation de son homosexualité l'y aurait conduit, alors que sa situation financière ne le lui permettait pas. Comme le rappelle Peter Robinson (2016 : 127), « expulsion from Bon Vivant [en français dans le texte] society is the fate that awaits those who misunderstand the rules and threaten violence to innocent parties ». Lear affirmerait ici à la fois son homosexualité, la connaissance de la règle selon laquelle il est souhaitable de n'en faire pas mention, et enfin de son choix de s'y tenir en ne la révélant qu'à travers un poème dont la valeur cryptique reste bien gardée par les possibilités interprétatives.

\subsection{Un poème absurde ?}

Nous avons montré dans Cusimano (2012) comment l'absurde se trouve condamné à ne rester qu'une couche de lecture : une lecture attentive ou mieux informée (par exemple biographique) ou même le simple fait que l'homme, en tant que machine interprétative, ne saurait jamais se contenter du non-sens, sont susceptibles de dissoudre l'absurde ou de le reléguer à l'état de couche de lecture sédimentée. Ce poème ne semble pas devoir déroger à la règle mais, contrairement à d'autres textes, n'a pas même été envisagé sous cet angle par les différents critiques et connaisseurs de Lear, quand bien même serait-il issu d'un ouvrage dont le titre est « nonsense », ce qui peut paraître une curiosité. Aussi cette section se veut-elle une réparation de cet oubli critique.

Passons rapidement sur le fait que les oignons ne sont qu'empruntés pour préparer la farce et sur le caractère grotesque de leur quête : en effet, tout d'abord cherchent-ils les ingrédients idoines dans les magasins (« shops ») puis au marché (« market ») et enfin, plus justement en leur qualité de pauvres, dans les jardins (« gardens »). De même, le lien entre perdre ses dents et ses sourcils (« lose our teeth and eyelashes », vers 8) et ne pas dîner (« if we haven't any dinner », vers 7) n'est pas franchement marqué du sceau de la logique. Autre chose encore : peut-on correctement se nourrir à deux avec une seule souris et un seul oignon?

Mais les plus nets soupçons doivent se concentrer sur l'homonymie entre sage1 ( « sauge » en français) et sage2 (« sage » en français »). Edward Lear aurait pu facilement désambiguïser les deux homonymes de manière typographique en écrivant Sage 2 avec une majuscule et non sage1 mais tous deux sont systématiquement orthographiés avec la majuscule, comme tous les autres protagonistes pourvus du trait /animé/ et les lieux/objets essentiels du poème. Deux options sont possibles pour les deux vieillards, dont l'une 
apparaît clairement contraire à la raison et au sens commun, absurde en un mot : car alors que 'sauge' (soit sage1) et 'oignons' forment une isotopie générique //alimentation//, 'sage' (soit sage2) et 'oignons' unis par le même coordonnant « et » forment au contraire une allotopie, c'est-à-dire une rupture de la récurrence sémique attendue et mettent en jeu des sèmes génériques qui s'opposent (presque) toujours : /humain/ vs. /alimentation/. Cette allotopie peut certes se trouver dissoute dans un contexte de cannibalisme, mais de prime abord ce n'est pas le cas ici : c'est plus par défaut et par une ignorance aussi coupable qu'invraisemblable que les deux vieillards se résolvent à produire cet effort d'ascension pour atteindre le sage.

La figure suivante résume ce qui vient d'être dit. Rappelons que très fréquemment, lorsqu'il y a coordination bisegmentale, c'est depuis le second élément que se propagent les sèmes : ici 'onion' valide 'sage1' par assimilation régressive et invalide 'sage2'.

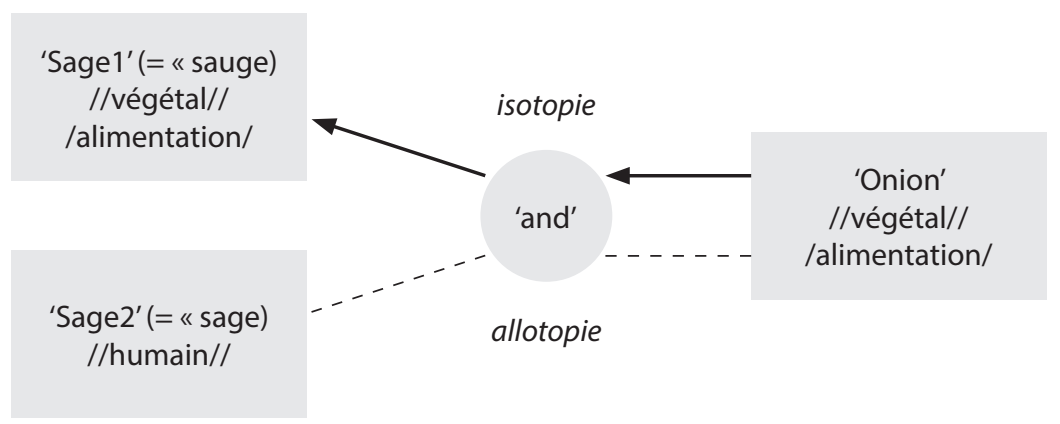

Comme le dit justement Peter Robinson (2016: 126), « The two old bachelors are fools, though, because not sagacious enough to appropriately disambiguate the sage that goes with onions in some stuffing from the kind of old fellow too wise to make such a category mistake ». Une erreur de catégorisation qui les conduit à leur perte et leur exclusion de la bonne société victorienne. Nous ferons aussi remarquer que cette méprise est présente dans le texte avant l'épisode du sage : 'souris', en tant que /nuisible/, n'est pas non plus pourvu du sème /alimentation/ et demeure sauf cas de famine non-comestible.

En un sens, la figure du sage elle-même, bien que Peter Levi le considère comme « the only forceful character » (illustrée par Lear et même la seule dont on distingue le visage), est douteuse, car on apprend que non seulement il ne fait que lire à longueur de journée, mais surtout toujours une même page : «who reads all day a most perplexing page ». Est-il vraiment le garde du savoir, de la connaissance, ou simplement une image de la société victorienne? 


\section{LA FIGURE DU BARDE DANS LA TRADUCTION DE PATRICK HERSANT}

Comme on a pu le voir, le mot « sage » se comporte comme la plaque tournante du poème, l'échangeur de deux interprétations incompatibles car il ne s'agit clairement pas de polysémie : la méprise sur l'homonymie et la narcotisation de l'interprétation la plus logique dans le contexte d'une coordination avec « onions » conduit les deux vieillards à une action non seulement absurde mais aussi vouée à l'échec. Peter Robinson (2016: 126) rappelle que ce genre d'erreurs peut être le fait de locuteurs insuffisamment rompus aux spécificités de la langue cible en traduction : « [you] (...) aren't able to disambiguate a dictionary definition - as when, in translating from another language, you find the word in your bilingual dictionary but don't understand the context enough to pick the appropriate meaning from the differentiated list ».

Patrick Hersant (1977 : 12-13) est au contraire un traducteur émérite et lorsqu'il forme le projet de traduire l'ensemble de l'œuvre de Edward Lear, il n'ignore aucune des difficultés auxquelles il sera confronté :

Entre une langue qui tourne à vide et un idiome nonsensique, mais neuf, Lear a tôt fait de choisir. La poésie victorienne réclame un traitement de choc. Or, dans la seconde moitié du XIXème siècle anglais, tout progrès dans le domaine des lettres semble devoir passer par un renouvellement du langage. On peut distinguer, avec Steiner, trois tentatives en ce sens : «Un processus de dislocation, l'amalgame de langues existantes ou la quête d'un système de néologismes ».

Dans ce texte, on trouvera bien un néologisme : « purpledicular » (dans lequel on reconnaît « purple » : « violet ») que Hersant traduit par « pierre-pendiculaires », préférant la conservation de la dimension néologique que celle du contenu sémantique.

Si sa traduction de «stuffin » par « paupiettes » ne semble pas poser de problème majeur, le muffin devient une banane, semble-t-il dans le seul but de marquer la rime avec « cabane » du vers 1 précédent. Mais à y regarder de plus près, il pourrait s'agir aussi de contrebalancer l'absence de figures phalliques dans la traduction. Cela pose toutefois un problème subsidiaire : la banane dans la période victorienne, on peut l'imaginer, devait être un fruit précieux et qui n'aurait donc pas sa place dans une maison de « fauchés », comme l'a écrit Hersant. Dans le même ordre d'idée, le « givre » de la traduction, fruit de l'imaginaire montagnard du traducteur, n'a pas de pendant dans le texte original et semble seulement sollicité pour la rime avec « livre ». Autre chose : plus de trace du citron et du miel, pourtant de parfait compagnons du thé, mais un « croûton de pain » plus français. Les oignons sont maintenus, tout comme le caractère générique de la recette est aussi bien marqué lexicalement, il nous semble, grâce à « découpez », " réservez », « entourez » et « prête ». La tripartition shops/market/gardens est par contre perdue au profit 
d'une bipartition marchés/jardins, ce qui ne rend pas justice à la décadence progressive de l'ambition des vieillards. Enfin, le texte de Hersant est tout au présent, quand la version originale est au prétérit.

Mais le choix le plus difficile de Patrick Hersant concerne évidemment « sage », intraduisible en français à cause de l'absence d'homonymie à une lettre et un phonème près entre « sauge » et « sage ». Celui-ci explique dans sa préface l'option suivie :

Le plus souvent, le discours du nonsense tente de supprimer la polysémie du langage naturel. Tels ces « Deux vieillards célibataires » qui, pour réaliser une recette de cuisine, se mettent en quête de « barde », mais le vieux Barde qu'ils trouveront en route par la vertu de son homonyme, leur suffira amplement. La désignation tient lieu d'expression, hors de toute signification.

Ainsi, « sage » se trouve dans la traduction remplacé par « barde » qui, d'après le Trésor de la Langue française en ligne se définit comme « [chez les Celtes] Poète-chanteur qui célébrait les exploits des héros en s'accompagnant de la cruth (sorte de lyre) ». Il n'est peut-être pas approprié de tenir rigueur à Hersant de la différence, toutefois notable, entre la figure du sage avisé et celle du barde-artiste, mais ce choix entraîne des conséquences importantes : par exemple, il est de fait plus délicat d'imaginer un barde lisant toute la journée un énorme livre plutôt que jouant d'un instrument. On connaît aussi la répugnance des Gaulois pour l'écriture, ce qui ne joue pas non plus en faveur de la lecture continue d'un livre, de surcroît énorme.

Ceci posé, nous dirons surtout que l'effet le plus ennuyeux de ce choix se propage jusqu'à l'homonyme de "barde » (poète-chanteur) : il nous a fallu chercher l'équivalent de sage1 (la sauge) dans la traduction française. Nous avons d'abord cherché une plante -inexistante, avant de nous raviser : selon le Dictionnaire de la langue françoise ancienne et moderne, Volume 1 de Pierre Richelet (1735), il s'agit d'une « tranche de lard, déliée \& large, dont on couvre quelquefois les poulets, les chapons, \&c., avant de les mettre à la broche ». Cela fait surgir trois nouveaux problèmes en cascade : « barde » dans ce sens, est féminin, comme l'indique sans ambages les exemples fournis par Richelet (« Une bonne barde, une barde trop large, trop mince. Levez une barde \& mettez-la sur ce chapon »), alors qu'on dit bien « un barde » lorsqu'on vise le poète-chanteur ${ }^{2}$ : Hersant a donc dû faire en sorte de neutraliser les contextes qui l'obligeraient à ajouter tant déterminants qu'adjectifs. Le second problème, le plus sérieux, a trait au fait que « barde » ne s'emploie plus en français contemporain, ce dont son atteste sa présence dans le TLFi accompagné d'un exemple datant de 1873, et, au contraire, sa présence dans le Dictionnaire de l'Académie française de 1814 qui en donne une définition très proche de celle que nous avons livrée plus haut : « aujourd'hui [en 1814 donc] ne se dit

2 Un tel problème ne se présenterait pas en anglais, langue dont le genre n'est pas marqué morphologiquement. 
plus que pour signifier une tranche de lard fort mince dont on enveloppe les chapons, des gélinottes, des cailles, et autres oiseaux, au lieu de les larder. Une barde de lard». Il s'agit donc bien plus encore que d'un usage vieilli, c'est un mot qui ne s'emploie plus du tout : comment, dans ce cas, l'homonymie pourrait-elle être dès lors perçue et ressentie par les lecteurs? En outre, effet moins grave sans doute, on a un basculement depuis un sème générique //végétal// contenu dans « sauge » vers le sème //animal// de « barde ». Hersant recourt alors à une solution simple pour désambiguïser les barde1 (le lard) et barde2 (le poète-chanteur), une option à laquelle Edward Lear s'était bien sûr refusé puisqu'il souhaitait justement conserver la non-différenciation des homonymes de toute autre manière que par le contexte : le traducteur utilise donc la minuscule initiale pour barde1 et la majuscule pour barde2.

Parmi les autres différences notables nous souhaitons aussi signaler celles qui sont particulièrement réussies : dans les deux couples de vers « Ils crient : « Ô Barde ! Assez lu ! Selon la recette / Tu finiras tes jours autour de nos paupiettes ! » et « Vexés, ils sont partis sans qu'on les ait revus, / Et nul ne sait, depuis, ce qu'ils sont devenus », le sens, le rythme comme le rime sont du plus bel effet. Mais à l'impossible nul n'est tenu : le signifiant « sage » en anglais, associés à deux signifiés tout à fait différents, n'offre aucune prise au traducteur français. Il est, au regard de ces observations, plutôt curieux que Patrick Hersant n'ait pas pris la peine de faire quelques précisions. Or il n'est pas impossible que le traducteur ait eu ses raisons que nous essayons de formuler ci-après.

\section{CONCLUSION}

La traduction de Patrick Hersant préserve certes la possibilité de l'interprétation générale privilégiée par la plupart des critiques, à savoir l'explication (homo)sexuelle. Mais elle opère des choix plus embarrassants en réduisant presque à néant, pour un locuteur contemporain, la construction du nonsense du poème telle que Edward Lear l'a conçue : la bascule qui a lieu grâce à l'homonymie de « sage » en anglais est laissée pour compte avec « barde ». Nous ne feignons pas d'ignorer la difficulté de la tâche et nous serions bien en peine de proposer une alternative traductologique. Mais à dire vrai, l'essentiel est ailleurs : Patrick Hersant, à travers sa traduction, laisse entendre que celle-ci doit être lue accompagnée de la version originale, idée confortée par la présentation même de son ouvrage. En effet, la traduction y est placée en miroir, à droite, systématiquement en correspondance exacte vers après vers. La figure du barde est posée comme une incitation à observer en résonance avec la version originale, seul juge de paix. C'est donc un choix de traducteur conscient de l'impossibilité de toute traduction satisfaisante qu'opère Patrick Hersant : ce faisant, il s'arrête dans sa démarche, en toute modestie, à mi-chemin et sa traduction semble simplement destinée à aider les lecteurs francophones dans leur saisie du poème de Edward Lear. La figure du barde, en tant 
que protagoniste insuffisamment complet car privé de son homonyme, est à mettre nécessairement en balance avec le sage/sauge de la version originale. S'inscrivant dans une longue tradition de traduction lâche de textes anglais vers le français, la traduction de Hersant offre une nouvelle illustration de la conception qui veut que toute traduction doive être perçue comme un autre texte.

\section{BIBLIOGRAPHIE}

BEVIS, Matthew (2016) Falling for Edward Lear. J. Williams et M. Bevis (dir.), Edward Lear and the play of poetry. Oxford : Oxford University Press, 134-161.

CUSIMANO, Christophe (2012) La sémantique contemporaine du sème au thème. Paris : Presses Universitaires de Paris-Sorbonne.

LEAR, Edward (1877) Laughable lyrics, a fourth book of nonsense poems, songs, botany, music, etc. Londres : Robert John Bush.

LEAR, Edward (1977) Nonsense. Traduction de Patrick Hersant. Toulouse : Ombres.

LEVI, Peter (2013) Edward Lear - A life. Londres : Tauris.

ROBINSON, Peter (2016) Edward Lear : celebrity chef. J. Williams et M. Bevis (dir.), Edward Lear and the play of poetry. Oxford : Oxford University Press, 115-133.

\section{ANNEXES}

\section{The two old bachelors}

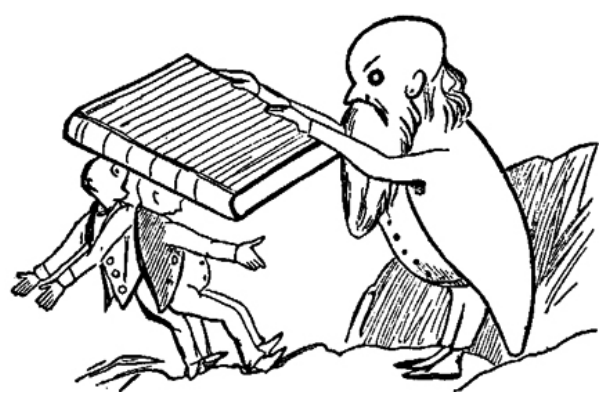

Two old Bachelors were living in one house;

One caught a Muffin, the other caught a Mouse.

Said he who caught the Muffin to him who caught the Mouse,--

'This happens just in time! For we've nothing in the house, 
'Save a tiny slice of lemon and a teaspoonful of honey,

'And what to do for dinner -- since we haven't any money?

'And what can we expect if we haven't any dinner,

'But to loose our teeth and eyelashes and keep on growing thinner?'

Said he who caught the Mouse to him who caught the Muffin,--

'We might cook this little Mouse, if we had only some Stuffin'!

'If we had but Sage and Onion we could do extremely well,

'But how to get that Stuffin' it is difficult to tell'--

Those two old Bachelors ran quickly to the town

And asked for Sage and Onions as they wandered up and down;

They borrowed two large Onions, but no Sage was to be found

In the Shops, or in the Market, or in all the Gardens round.

But some one said, -- 'A hill there is, a little to the north,

'And to its purpledicular top a narrow way leads forth;--

'And there among the rugged rocks abides an ancient Sage,--

'An earnest Man, who reads all day a most perplexing page.

'Climb up, and seize him by the toes! -- all studious as he sits,--

'And pull him down, -- and chop him into endless little bits!

'Then mix him with your Onion, (cut up likewise into Scraps,)--

'When your Stuffin' will be ready -- and very good: perhaps.'

Those two old Bachelors without loss of time

The nearly purpledicular crags at once began to climb;

And at the top, among the rocks, all seated in a nook,

They saw that Sage, a reading of a most enormous book.

'You earnest Sage!' aloud they cried, 'your book you've read enough in!--

'We wish to chop you into bits to mix you into Stuffin'!'--

But that old Sage looked calmly up, and with his awful book,

At those two Bachelors' bald heads a certain aim he took;--

and over crag and precipice they rolled promiscuous down,--

At once they rolled, and never stopped in lane or field or town,--

And when they reached their house, they found (besides their want

$$
\text { of Stuffin',) }
$$

The Mouse had fled; -- and, previously, had eaten up the Muffin.

They left their home in silence by the once convivial door.

And from that hour those Bachelors were never heard of more. 


\section{Deux vieillards célibataires (traduction de Patrick Hersant)}

Deux vieillards célibataires dans une cabane ;

L'un attrape une souris, l'autre une banane.

Ce dernier au premier dit :

«Voilà qui tombe à merveille : au garde-manger,

Il ne reste guère plus qu'un croûton de pain.

Que mangerons-nous ce soir? Nous sommes sans un!

Or, si nous devons passer la soirée à jeûn,

Nous perdrons nos dents, nos cils - et notre embonpoint ! »

À quoi l'autre vieillard dit : « En vain tu t'inquiètes !

«Nous avons une souris: faisons des paupiettes!

De l'oignon, un peu de barde, ça devrait marcher -

Hélas, le problème est là : nous sommes fauchés!»

Alors les deux vieux garçons sortent en courant

Et quêtent dans le village ces deux ingrédients.

Ils trouvent bientôt l'oignon, mais de barde, point :

Ils écument les marchés, les jardins - en vain!

Enfin on leur dit : «Là-bas, parmi les sommets

Pierre-pendiculaires, est un sinueux sentier

Menant à une caverne : là médite un Barde -

Un poète illuminé portant une barbe.

Profitant de sa stupeur, jetez-vous sur lui :

Découpez-le en lanières, réservez; et puis, Ayant farci d'un oignon votre souricette,

Entourez de votre Barde ; la paupiette est prête ! »

Alors les deux vieillards célibataires

Grimpent aux sommets pierre-pendiculaires.

Là, parmi les rochers noirs brisés de givre,

Ils aperçoivent leur Barde, plongé dans un livre.

Ils crient : «Ô Barde, Assez lu ! Selon la recette,

Tu finiras tes jours autour de nos paupiettes!»

Or, c'était maladroit. Le Barde, calmement,

Assomme les vieillards de son tome imposant ;

Ceux-ci roulent alors de ravin en ravine.,

Dévalant les coteaux, dévalant les collines -

Les voilà qui atteignent, épuisés, leur cabane :

La souris s'est enfuie, emportant la banane.

Vexés, ils sont partis sans qu'on les ait revus,

Et nul ne sait, depuis, ce qu'ils sont devenus. 
POVZETEK

\section{»The Two Old Bachelors« Edwarda Leara: pomenoslovna in prevodoslovna analiza}

Namen pričujočega prispevka je pomenoslovna obravnava kratkega poetičnega besedila z naslovom »The Two Old Bachelors«, ki ga je Edward Lear leta 1877 objavil v svoji zbirki Laughable lyrics, a fourth book of nonsense poems, songs, botany, music, etc. Natanko sto let po izidu je pesem v francoščino prevedel Patrick Hersant (Edward Lear: Nonsense, 1977). V prispevku predstavljamo pomenoslovno analizo izvirne različice pesmi v angleščini in ponujamo razmislek o tem, zakaj lahko pesem uvrščamo med literarna dela absurda. Na podlagi pripravljalne študije pokažemo, kako je Hersant razrešil dilemo, s katero se je soočil pri prevajanju Learove pesmi: eden izmed elementov absurda v pesmi namreč temelji na enakozvočju samostalnika sage, ki je angleški izraz za žajbelj, in samostalnika sage, ki v angleščini poimenuje modreca. Osebi v pesmi žajbelj potrebujeta za pripravo nadeva; namesto žajblja najdeta modreca, ta pa ju brez besed prepodi z gore, na kateri sedi. To dejanje lahko razumemo kot simbol nevarnosti, ki je med viktorijansko dobo pretila istospolno usmerjenim osebam. Hersant se je prevajalskega izziva lotil tako, da je ustvaril različico, ki z izvirnikom nima veliko skupnega. Če upoštevamo dolgo tradicijo prostega prevajanja iz angleščine $v$ francoščino, lahko torej na njegov prevod gledamo kot na še en primer dojemanja prevoda kot povsem novega besedila.

Ključne besede: Edward Lear, Two Old Bachelors, viktorijanska doba, nesmisel, absurd, pomenoslovje, prevodoslovje, enakozvočje, Hersant

\section{ABSTRACT}

\section{"The Two Old Bachelors" by Edward Lear: a semantic and traductological analysis}

The aim of this article is to describe from a semantic point of view Edward Lear's poetic short text entitled "The Two Old Bachelors" (from Laughable lyrics, a fourth book of nonsense poems, songs, botany, music, etc., 1877) translated into French exactly a century after it was published by Patrick Hersant (Edward Lear - Nonsense, 1977). In addition to a properly semantic analysis of the original English version of the poem and what makes it an absurd text, we wish to show on the basis of this preparatory study how Patrick Hersant addressed the traductological dilemma he was confronted with: indeed, a part of the absurd aspect of the text is based on the homonymy of "sage" in English, which can be a plant (sage1) or a wise man (sage2). While they need the first one to make their recipe for stuffing, they find as a substitute a clever man who quietly ejects them from the mountain where he sits. It can be seen as dealing with the threat faced by homosexuals during the Victorian period. Confronted with this difficulty, the translator delivered an interpretation that is very little faithful to the original. As part of a long tradition of loose translation from English 
to French, Hersant's text offers a new illustration of the concept that any translation should be perceived as another text.

Keywords: Edward Lear; Two Old Bachelors; Victorian; nonsense; absurd; semantics; traductology; homonymy; Hersant 